\title{
Landmark geometry and identity controls spatial navigation in rats
}

\author{
CORREIGH M. GREENE and ROBERT G. COOK \\ Tufts University, Medford, Massachusetts
}

\begin{abstract}
In three experiments, a new reference memory procedure was used to examine how male rats search for consistently located food in a cue-controlled spatial environment. The animals searched the tops of 24 poles for six hidden baits in an enclosed circular arena containing a fixed configuration of six object landmarks. In Experiment 1, acquisition was faster and overall performance better for the consistent group ( 10 rats), in which the six baited poles were fixed relative to the landmarks for each session, than for the random group (4 rats), in which baited poles were randomly configured. Cue-control tests and computer simulations suggested that the consistent group relied on the landmarks to directly go to the baited poles, whereas the random group used them to employ a response strategy for searching the arena. Experiments 2 and 3 revealed that the number, identity, and geometric configuration of the landmarks were important to the consistent group's search performance. Overall, these results are most consistent with the use of a geometric representation by male rats which includes information about both the identity and the relative geometry of discrete landmarks in the surrounding spatial environment.
\end{abstract}

In searching for food, many animals demonstrate an accurate working knowledge of their surrounding environment. Two general questions have been of interest in the investigation of this spatial behavior: What stimuli in the environment govern this behavior, and how are these stimuli encoded and used by animals for navigation? Although research addressing these basic questions has involved many species, including bees (Cartwright \& Collett, 1983; Cheng, Collett, Pickhard, \& Wehner, 1987; Gould, 1986), pigeons (Cheng, 1989, 1994; Spetch \& Edwards, 1988), hamsters (Poucet, Chapuis, Durup, \& Thinus-Blanc, 1986), gerbils (Collett, Cartwright, \& Smith, 1986), and chimpanzees (Menzel,1973; Tinklepaugh, 1932), by far the most popular approach has been to look at the behavior of rats in laboratory spatial discrimination tasks (see the review by Leonard \& McNaughton, 1990). Interestingly, the cognitive mechanisms underlying rat spatial behavior are still a source of considerable disagreement. We think that a primary reason for this state of affairs has been a frequent failure to control and examine the complex spatial and visual cues employed in most of these spatial tasks (Cook, 1993).

Consider the widely used radial maze task as an illustration of this problem. In the radial maze, rats search for hidden food located at the end of eight arms that radiate out from a central platform (Olton \& Samuelson, 1976).

The preparation of this report was supported by a grant from the National Science Foundation to R.G.C. The research was also partially supported by a Faculty Research Award from Tufts University. The authors thank Jane Anderson, Jeffrey Katz, Brian Cavoto, Kimberley Cavoto, and Matthew Schlesinger for their helpful suggestions on earlier drafts. Requests for reprints should be sent to R. G. Cook, Department of Psychology, Tufts University, Medford, MA 02155 (email: rcookl@emerald.tufts.edu).
Rats perform this task exceedingly well on the basis of extra-maze cues in the surrounding laboratory room (Kraemer, Gilbert, \& Innis, 1983; Olton, 1978; Zoladek \& Roberts, 1978). Debate continues, however, about how these extra-maze cues are encoded and used by rats. Are these extra-maze cues globally integrated into a configural representation of the room's space in which the relations among the cues are retained, or instead, are the different cues encoded separately and independently from one another and used more like series of places to visit?

Suzuki, Augerinos, and Black (1980) conducted one of the most widely cited and revealing radial maze studies done on this issue so far. Using a surrounding environment that consisted of seven extra-maze cues within a curtained area, they found that transpositions of the cues, but not simple rotations, disrupted the rats' performance. These findings suggested that the rats had encoded the spatial or visual relations among the cues while performing the task, which is consistent with a map-like configural encoding hypothesis. Other research has suggested, however, that such cue relations are not always encoded in spatial tasks. For instance, using a reference memory procedure, McNaughton, Elkins, and Meltzer (unpublished data described in Leonard \& McNaughton, 1990) analyzed which of several cues were used by rats to locate a repeatedly baited arm in a radial maze. They found that the rats relied primarily upon the geometrical relation between only one extra-maze cue and the baited arm, which suggested that the rats were not using the entire configuration of spatial cues (see also Tolman, Ritchie, \& Kalish, 1946). Using a computational approach, Brown (1992) found that rat radial maze performance could be accounted for by a Markov choice model, in which the individual arms were treated as spatially unrelated independent entities. The latter studies are thus more consistent with an independent 
cues model of landmark use. Part of the reason why the debate continues between these positions is that in most radial maze studies, the critical extra-maze cues are usually just the uncontrolled contents of a standard laboratory room (e.g., Cook \& Brown, 1985; Cook, Brown, \& Riley, 1985). Without explicit control over the spatial environment these configural and independent cue alternatives are essentially indistinguishable.

The experiments reported in this article represent an attempt to correct this problem by exploring a promising new open field procedure for testing rat spatial behavior-one that allows greater experimenter control over the critical spatial cues and more precise measurement of the animal's choice behavior. Fourteen rats were tested in a large enclosed circular arena that contained twentyfour $15-\mathrm{cm}$ poles (see Figure 1). The rats' daily task was to find the six poles that were baited with food hidden in small cups on top of each pole. To guide this search, six landmarks were placed about the arena's surface. These landmarks were the only reliable cues to the hidden food, because other potential extra- or intra-arena cues were rendered useless. This was accomplished by placing a uniform opaque curtain completely around the arena to prevent the rats from seeing the extra-arena environment and by rotations of the arena relative to the landmarks and the external laboratory room. We conducted three experiments using a reference memory procedure to investigate how the landmarks in the arena would be employed by the rats to locate the hidden food.

\section{EXPERIMENT 1}

We examined the acquisition of this new spatial task by two groups of rats in Experiment 1. For the consistent group, the six baited poles were consistently located in relation to the landmarks on every daily trial, thus permitting the landmarks to serve as spatial cues to the hidden food. For the random group, the six baited poles were quasi-randomly relocated in relation to the landmarks on every trial. The random group served four functions: (1) it helped evaluate whether the food's odor could guide pole choice, (2) it permitted the evaluation of how search or response strategies (e.g., avoiding recently chosen poles) contributed to performance, (3) it provided a reference for judging the effectiveness of the consistent group's performance, and (4) it helped, in conjunction with Monte Carlo simulations, to establish what "chance" performance was in the task.

After the rats had learned the task, three tests were conducted to evaluate what cues controlled each group's performance. In the first test, we investigated the contribution of nonvisual extra-arena cues (e.g., localized sounds or smells) by examining performance with novel orientations of the arena within the laboratory room. In the second test, we assessed the importance of the landmarks by examining performance when they were removed. The third test was an investigation of how the rats performed when they started from novel locations within the arena.

\section{Method}

\section{Subjects}

The subjects were 14 male Long-Evans rats individually housed on a 12:12-h light:dark cycle. They were maintained at $85 \%$ of their age-adjusted free feeding weights during the experiment, with free access to water in their home cages. Any supplemental feeding occurred after each daily session.

\section{Apparatus}

The testing arena was a $167-\mathrm{cm}$-diameter circular platform surrounded by a sheet metal wall $25 \mathrm{~cm}$ in height. We surfaced the

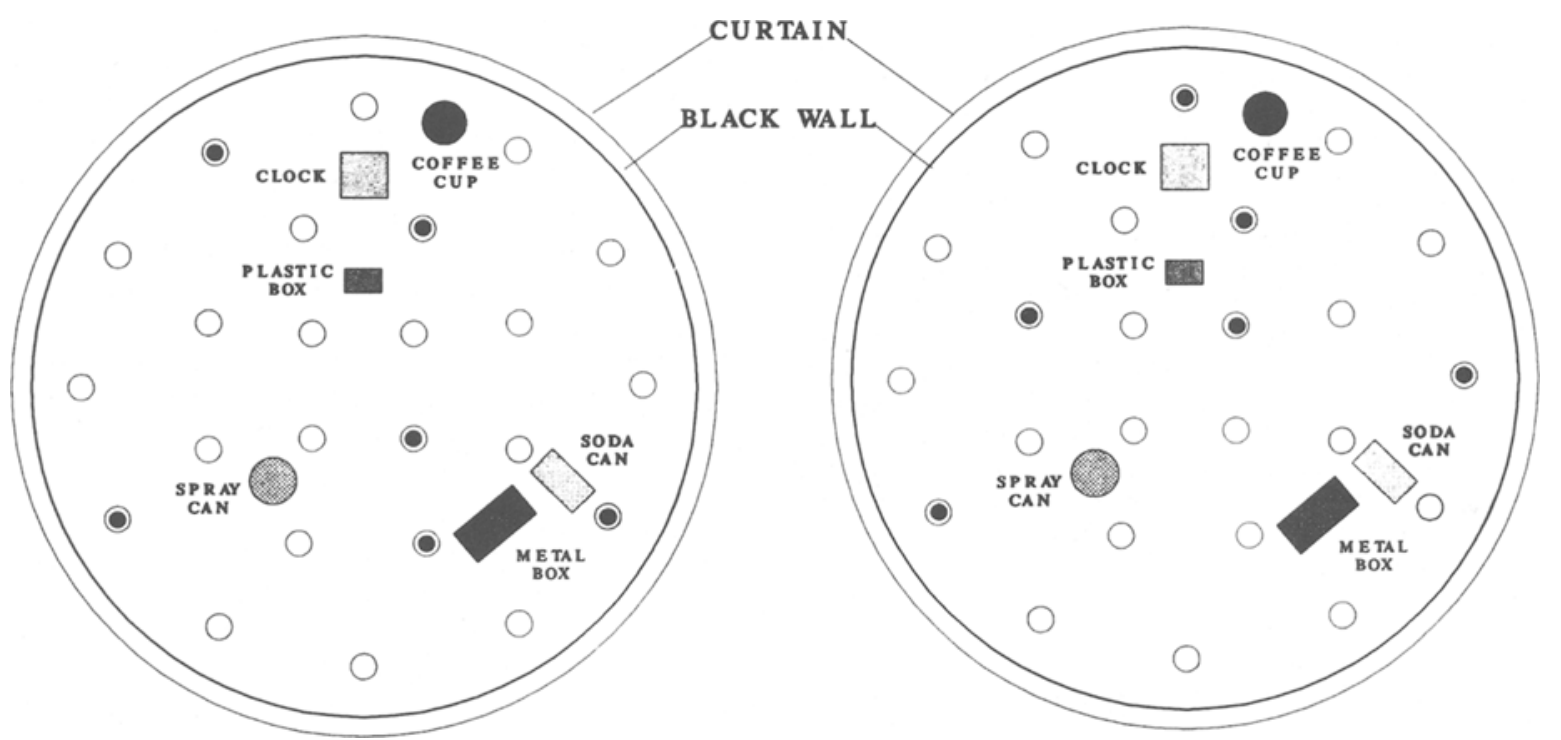

Figure 1. Overhead views of the two arena configurations used with two sets of subjects (5 rats each) in the consistent group. Depicted for the two configurations are the positions of the six landmarks, baited poles (darkened circles), and unbaited poles (open circles). 
wooden floor of the testing arena with smoothed plaster to cover the oriented grain of the wood and fill any distinctive holes. The entire apparatus was painted flat black. The arena was elevated $89 \mathrm{~cm}$ off the floor and mounted on a rotating base that allowed it to be moved freely to any orientation with respect to the room. Illumination was provided with a square bank of fluorescent lights that was $157 \mathrm{~cm}$ above the center of the testing arena. We completely enclosed the arena within a uniform brown paper curtain suspended from the ceiling. Four small openings (located at $90^{\circ}$ intervals around the arena) in the curtain allowed the recording of the sequence and timing of pole choices by the rats with a 24switch event recorder interfaced to an AT-class microcomputer.

Twenty-four wooden poles ( $15 \mathrm{~cm}$ tall; $1.9 \mathrm{~cm}$ in diameter) were positioned in three concentric circles within the arena (see Figure 1). The inner ring ( $38 \mathrm{~cm}$ in diameter) consisted of 4 poles, the middle ring ( $85 \mathrm{~cm}$ in diameter) consisted of 8 poles, and the outer ring ( $138 \mathrm{~cm}$ in diameter) consisted of 12 poles. We selected this pole configuration to best equate the distances between adjacent poles both within and between rings (mean interpole distance $=30 \mathrm{~cm}$; range of $26-33 \mathrm{~cm})$. A small brass food cup $(2.5 \mathrm{~cm}$ in diameter and $1 \mathrm{~cm}$ deep) capped each pole. Six distinctive objects served as landmarks within the arena. These consisted of a weighted pink and white soda can ( $12 \mathrm{~cm}$ in height $\times 6 \mathrm{~cm}$ in diameter) lying on its side, a metal feeding cup for pigeons $(10 \times 8 \times 7 \mathrm{~cm})$, a spray paint can $(20 \times 7 \mathrm{~cm})$, a plastic slide box $(6 \times 5 \times 5 \mathrm{~cm})$, a brown digital clock (trapezoidal in shape; $7 \times 7 \times 6 \mathrm{~cm}$ ), and an inverted white and blue coffee cup ( $9 \mathrm{~cm}$ in height, $8 \mathrm{~cm}$ in diameter).

\section{Procedure}

Experimental conditions. The 10 rats in the consistent group received one of two fixed configurations of baited poles relative to the six landmarks. Five rats were tested with each configuration. These two configurations always had three food locations in the outer ring, two in the middle, and one in the inner ring of poles (see Figure 1). Other than this distributional constraint, these two configurations of food locations were generated randomly and independently of the landmark locations. In contrast to the consistent group's stable landmark/food configuration, the 4 rats in the random group received a brand new configuration of baited pole locations each session. Each of these new configurations was randomly chosen, except for the three-two-one distributional constraint.

Task acquisition. We conducted two pretraining sessions to familiarize the rats with the testing environment and climbing the poles for food. In the first session, we released a squad of 3 or 4 rats together into the arena. For $10 \mathrm{~min}$, each squad was allowed to retrieve visible food pellets placed about the arena's surface and the top of the poles (food cups removed). For the next $5 \mathrm{~min}$, we replaced pellets only on top of the poles. In the second session, we added the food cups to the top of the poles and placed a single pellet on all 24 poles. Rats were individually tested in the arena. They were allowed to retrieve food for $20 \mathrm{~min}$ or until all poles had been visited. Discrimination training began with the next session.

Discrimination training. Over the next 48 discrimination training sessions, we baited only six of the poles per session in accordance with a rat's assigned condition. Each daily session consisted of one trial for each rat. Each trial started with the rat being released at the edge of the arena and ended when all six baited poles had been visited or $20 \mathrm{~min}$ had elapsed. We recorded a pole choice when a rat reared up to within $3 \mathrm{~cm}$ of the food cup. This choice behavior was obvious and easy to detect. The number of pellets placed in the baited food cups was gradually increased over training, beginning with two pellets on the 16 th session and three on the 30 th session. This was done to increase the motivation of the rats, while ensuring that they always consumed all pellets on each correct choice.

To eliminate potential cues other than the landmarks, we implemented a series of arena rotations on a regular basis throughout training and testing. During discrimination training, the arena was rotated daily between one of two orientations in the laboratory room $\left(0^{\circ}\right.$ and $90^{\circ}$ relative to a fixed reference point in the room). To eliminate intra-arena cues as a potential source of information about food location, we rotated the landmarks around the arena on a weekly basis $\left(0^{\circ}, 90^{\circ}, 180^{\circ}\right.$, and $270^{\circ}$ relative to a fixed arena reference point). Independently of the two former rotations, we used three different starting locations during training $\left(0^{\circ}, 45^{\circ}\right.$, and $90^{\circ}$ from a fixed reference point in the arena) which were randomly ordered within blocks of three sessions. Following 48 sessions of discrimination training, we conducted three cue control tests to evaluate the factors controlling the rats' ability to locate the baited poles in the arena.

Cue Control Test 1: Extra-arena cues. This test assessed whether cues external to the enclosed arena affected performance. In each of two daily (morning and afternoon) sessions, we tested rats either at one of the arena orientations used during training or at a novel orientation. We conducted two 4-day test blocks. The first 4-day block tested the $90^{\circ}$ (baseline) and $270^{\circ}$ (novel) orientations once each day, and the second 4-day block tested the $0^{\circ}$ (baseline) and $180^{\circ}$ (novel) arena orientations. The order of these test conditions within a day was randomized for each rat. Immediately after this test was completed (and for all remaining experiments in this paper), the arena's orientation within the room was randomly selected on a daily basis from among these four orientations

Cue Control Test 2: Landmark removal. This test examined the rats' performance when all of the landmarks were removed from the arena. During one day, we conducted two experimental sessions: in one, we removed all landmarks from the arena; in the second, the landmarks remained in their normal positions. The order of these test conditions was randomized for each rat.

Cue Control Test 3: Novel starting locations. The final test examined how a novel starting location influenced search performance. Rats were tested twice daily for 12 days. On each day, they were placed at the $45^{\circ}$ (baseline) starting location during one session and at the $225^{\circ}$ (novel) starting location in the other session. The order of these test conditions within a day was randomized for each rat.

\section{Results and Discussion}

In brief, the results of Experiment 1 suggested that we had developed a successful procedure for studying landmark-guided choice behavior in rats. Analyses of several measures of performance revealed that the consistent group learned the task faster and with greater accuracy than did the random group. The cue-control tests indicated that the landmarks were critical to the successful search performance of the consistent group. Interestingly, the random group's performance by some measures also improved with experience to levels greater than expected by chance. Although not able to use the landmarks to directly guide their search for their randomly hidden food, these rats appeared to learn how to avoid recently chosen poles by using cues in the arena.

\section{Task Acquisition}

Both groups' abilities to locate the baited poles improved over the 48 training sessions. As expected, the consistent group showed a far greater improvement and higher level of performance than did the random group. Figure 2 shows each group's choice behavior in six eight-session blocks as measured by three dependent variables: (1) total number of choices per session (upper panel), (2) number of correct poles in the first six choices (middle panel), and 
(3) choice efficiency (lower panel). The top panel shows that with training both groups reduced the number of choices needed to clear the arena of food, with the consistent group requiring markedly fewer choices than did the random group. The middle panel shows that the consistent group also improved over training in terms of the number of correct poles chosen within the first six choices of each trial (for details on this measure, see Olton \& Samuelson, 1976). The random group showed no improvement by this measure and remained at the same level of performance throughout the entire course of training.

The bottom panel displays a measure of performance that we termed choice efficiency. Choice efficiency is a very useful measure of performance in this task. It is derived by summing the serial positions of the six correct choices and dividing by the number of correct choices, resulting in the average sequential position of the six correct choices in each trial. For instance, if a rat's first six
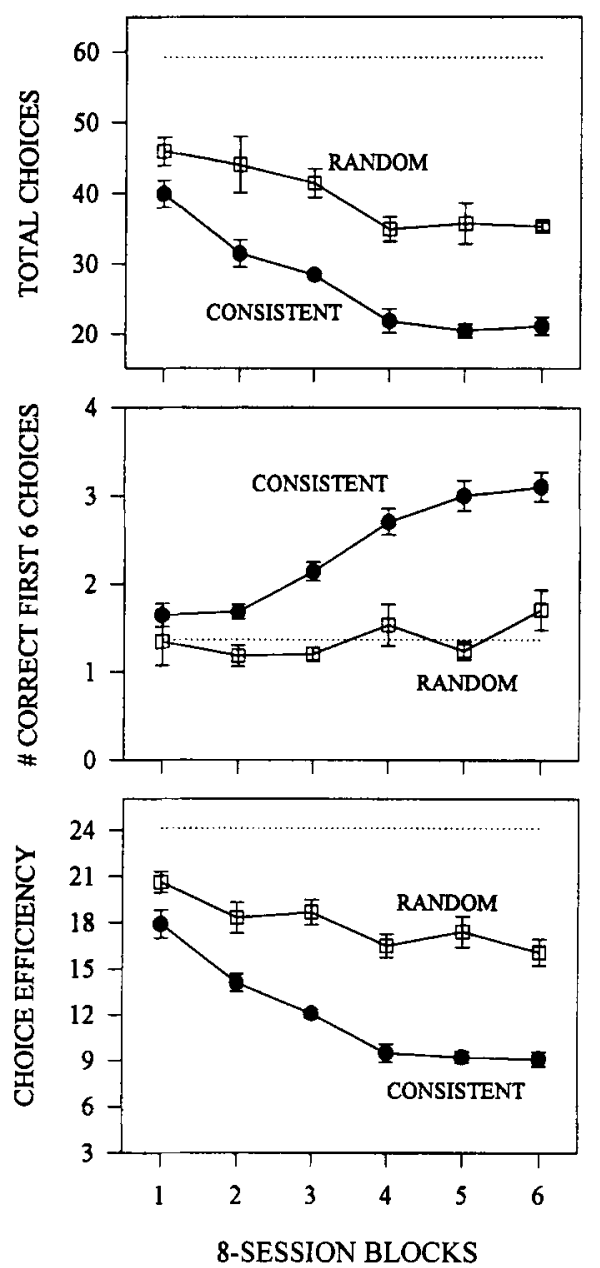

Figure 2. Mean performance for consistent and random groups in Experiment 1 over successive eight-session blocks, in terms of the total number of choices required to complete each session (top panel), the number of correct poles visited in the first six choices of a session (middle panel), and choice efficiency (bottom panel; see text for details). The dotted lines represent chance estimates for each measure as derived from a Monte Carlo simulation of performance. choices were all correct, its choice efficiency score would be $3.5[(1+2+3+4+5+6) / 6$; the best possible score $]$. As performance departs from this optimum, choice efficiency increases in value, as correct pole choices occur later in the overall sequence of choices [e.g., $(1+3+4+7$ $+9+11) / 6=5.8$ ]. Unlike the total choice measure, choice efficiency takes into account when in the search sequence correct choices occurred. Unlike the accuracy of the first-six-choice measure, it includes data from all choices, not from just a few. Consequently, it seemed the best way to capture both the sequencing and accuracy of all choices made by a rat within a trial. Like the two more traditional measures of spatial performance, choice efficiency also showed improvement over time, with the consistent group's performance again markedly superior to that of the random group.

We conducted repeated measures analyses of variance (ANOVAs), using each measure to examine changes in each group's performance over the experiment. All statistical tests were evaluated using an alpha level of $p \leq$ 05 . These found that the consistent group's choice accuracy significantly improved by all three measures [total choices, $F(5,45)=27.0$; number correct in the first six choices, $F(5,45)=24.1$; choice efficiency, $F(5,45)=$ 41.5]. The random group also showed significant performance improvements in choice efficiency $[F(5,15)=$ $3.9]$ and total choices $[F(5,15)=3.7]$, but not in the number correct in the first six choices $[F(5,15)=2.1]$. The latter finding is important, because it indicates that the smell of the food was not a factor in locating the baited poles. If these rats could have smelled the food over any distance in the arena, they should have proceeded directly to the baited poles. They did not.

Performance of the two groups was then directly compared using different mixed design ANOVAs (group $\times$ eight-session blocks) of each measure. The consistent group's faster acquisition of the task was confirmed by the presence of significant group $\times$ block interactions for both choice efficiency $[F(5,60)=4.1]$ and number correct in the first six choices $[F(5,60)=5.6]$. The analysis of total choices revealed only a significant main effect of group $[F(1,12)=118]$. The group $\times$ block interaction $[F(5,60)=1.3]$ was not significant for this measure.

\section{Estimation of Chance Performance}

While these results indicate that the consistent group outperformed the random group, they do not reveal how well the consistent group performed or help explain the random group's improvement, primarily because they do not include an estimate of chance performance. Given the procedure's open-field character, distributed multiple goals, and numerous choice opportunities, however, any direct computation of chance performance is difficult. Consequently, a Monte Carlo computer model was used to estimate chance performance. Within each simulated trial $(n=5,000)$, the program simply chose poles at random (with replacement) from a set of 24 imaginary poles, 6 of which were "baited" in exactly the same way as during a real trial. Each simulated trial ended when all 
6 baited poles had been found. The estimates of chance performance for the three measures based on these simulations were: total choices, 59.2 ; number correct in the first 6 choices, 1.36; choice efficiency, 24.1 (the dotted reference lines in Figure 2). Single mean $t$ tests comparing performance from the last eight sessions of training with these estimates confirmed that both groups were performing significantly better than these values. The sole and important exception was for number correct in the first six choices in the random condition, which was not significantly different from the chance estimate for this measure.

\section{Modeling the Random Group's Performance}

While the consistent group did take advantage of its stable landmark-food relations across sessions to markedly outperform the random group, the latter's performance did improve with experience in the arena. Because the random group's first six choices in the arena never exceeded chance expectation during the experiment, their increased search efficiency as detected by the other measures seems to reflect knowledge that must have been acquired within each session. We believe that this group gradually learned to avoid recently visited portions of the arena within each session in order to better its chances of finding the randomly located food hidden in the remaining portions of the arena. Visual analyses of the search paths taken by individual rats of the random group, however, revealed no easily identified consistent response or search strategy.

The Monte Carlo computer simulation used to estimate chance performance was particularly useful at this point. In the earlier simulation, any of the 24 poles in the arena could have been potentially chosen on any given choice. In reality, successive pole choices by the rats were frequently only within the near vicinity of the immediately preceding choice. Therefore, we conducted new simulations that more realistically reflected this aspect of their choice behavior. We added an algorithm that restricted otherwise "random" choices to the contiguous neighborhood of nearby poles following each choice (several rules for spatially restricting and relaxing these choices were explored; all produced essentially similar results). We additionally modified the program to "remember" a fixed number of recent choices, with each new choice replacing the oldest item in this "memory" buffer. By consulting this "memory" the program could further restrict the poles available for its next "random" choice among the nearby poles. This "memory" component can be thought of in two ways. It could reflect a true working memory for preceding pole choices in the arena. Alternatively, it could simply reflect the fact that rats appear to have an innate tendency not to repeat choices (see Olton \& Schlosberg, 1978). Thus, they might be able to eliminate portions of the arena without remembering specific locations simply by consistently shifting to new places in the arena.

Simulations with "memories" of different sizes in this neighborhood-restricted random choice model revealed the following results. First, the random group's performance during the first two blocks of training could be closely approximated by assuming that the last 5 visited poles were not involved in what was otherwise a "random" choice process. This 5-item "memory" capacity produced scores of $49.75,1.45$, and 20.30 for total choices, number correct in the first six choices, and choice efficiency measures, respectively. For the last two blocks of training, the model's best fit indicated an increase in "memory" to about 12 poles, resulting in scores of 37.5 , 1.5 , and 16.3 for the three measures above. Given these estimates, we suspect that early in training the random group was simply choosing at random from among the poles immediately in front of them as they moved about the arena. The "memory" estimate of 5 is close to number of poles that would be spatially behind a rat at most places in the arena. Later in training, however, these rats appear to have learned to be more systematic in their search by avoiding much larger portions of the arena after searching there. One final note from these various types of random choice simulations concerns the consistent group. Even random choice simulations involving "memories" for all 24 poles could not duplicate the consistent group's level of performance at the end of training, providing further evidence of this group's direct navigation to the baited poles.

\section{Cue Control Tests}

The overall superiority of the consistent group indicates that it had access to information about the food's location that was not available to the random group. The obvious source of this information was the stable spatial relations between food locations and the arena's landmarks, although other sources of information such as extra-arena cues or response strategies could have potentially been used also. These possibilities were all examined in the cue-control tests. Because the previous analyses indicated our three measures all captured the same basic effects both within and between the two groups, we primarily report future analyses of performance in terms of choice efficiency. This is done both for the purpose of brevity and because we viewed choice efficiency as the best of the three measures.

Novel orientations of the arena within the room performed in Cue Control Test 1 did affect the consistent group's accuracy on the 1st test day, but this quickly disappeared as performance returned to baseline levels. An ANOVA of the consistent group's efficiency (arena orientation $\times$ test block $\times$ session) revealed a significant interaction between the arena orientation and session $[F(3,27)=6.7]$. This interaction was due to a decline in the consistent group's choice efficiency during the first session with a novel orientation. $T$ tests comparing performance from the baseline and novel orientation sessions of each test block revealed that the only significant choice efficiency difference occurred on the first test block [choice efficiency for baseline $=8.2$ and novel orientations $=14.5 ; t(9)=5.6]$ and disappeared in sub- 
sequent blocks [mean efficiency over last three blocks: baseline $=9.9$ and novel orientations $=10.8$; all three $t \mathrm{~s}(9)<1.6]$. This brief decrement in performance suggests that the rats did possess some information about the maze's orientation relative to the room, perhaps from either visual information obtained as they were moved from their holding cages to the arena for testing or the internal maintenance of a generalized inertial heading.

Landmark removal in Cue Control Test 2 significantly reduced the effectiveness of the consistent group's search behavior. Mean choice efficiency significantly declined from 9.8 for the baseline session to 13.2 in the session when the landmarks were absent from the arena $[F(1,9)=$ 4.83]. The random group showed no significant difference in its choice efficiency between the baseline (14.0) and landmark-absent conditions $(16.2)$ sessions $[F(1,3)<$ 1.0]. A between-groups ANOVA comparing the consistent and random group's choice efficiency from the landmark-absent condition revealed no significant difference in performance between these groups $[F(1,12)<1.0]$, indicating that the consistent group's performance when the landmarks were removed dropped to the same level as the random group's.

The novel starting location introduced in Cue Control Test 3 did reliably lower the performance of the consistent group relative to the training baseline. An ANOVA (starting location $\times$ four-session blocks) comparing the consistent group's choice efficiency from the two different starting locations was significant $[F(1,9)=20.32]$. This difference overall was small (mean choice efficiency: $45^{\circ}=9.6,225^{\circ}=10.6$ ), and it did not significantly change over the three test blocks $[F(2,18) \leq 1]$. No similar effect was found on the random group's performance.

In sum, the removal of the landmarks caused the greatest disruption in performance for the consistent group, further evidence of their reliance on them for direct navigation. Changes to potential extra-arena cues had little or no sustained influence on their pole choices, suggesting that the different rotations and curtain had been generally effective in controlling for sources of spatial information from outside the arena.

Although the control exerted by the landmarks seemed to be the primary source of information, some responsebased factor may also have been involved to an extent in this group's search behavior. When tested from a novel release point in the arena, this group showed a very small, but reliable, reduction in their search efficiency. While the consistent group's performance from the novel release point was far better than chance estimates or that typical of the random group, this decrement suggests that some aspect of performance was tied to the three trained release points.

Using a water maze, a task in many ways similar to the present one, Morris (1981) previously reported that changing the starting locations of a consistent platform group had little influence on rat spatial behavior, with the animals taking "direct" paths to the hidden platform. This was especially true in comparison with conditions in which the platform was moved to a novel location in the pool (analogous to our random group's task). Such results suggest that swimming to the hidden platform was not based on learned specific routes, but was spatially guided by extra-maze cues. Pearce (1987) has strongly questioned the "directness" of these search paths in the water maze, because the rats' initial headings are often not toward the hidden platform. This disagreement stems directly from one of the greatest disadvantages of the water maze. It is very difficult to record when an animal actually is making a wrong "choice" response (e.g., reaching underwater for a nonexistent platform), so only successful performance can be judged easily. In comparison, our procedure permits the precise and direct measure of when and where choice errors occurred. As such, our novel starting location results suggest that a limited form of route-specific learning may take place in open-field tests more often than previously suspected.

We added new release points $\left(135^{\circ}, 315^{\circ}\right.$, and center $)$ to the rats' daily training regimen in the weeks following this experiment. Interestingly, these new locations produced little or no decrement in choice accuracy upon their introduction. This later complete transfer to novel release points indicates generalized control of search behavior by the landmarks. Maybe the rats had stopped using any response strategy in the interim because of the greater number and variety of starting locations experienced after the addition of the $225^{\circ}$ release location to their regular training schedule. Nevertheless, the more cautious interpretation of the earlier data is that some form of response-based strategy may have initially contributed to the consistent group's landmark-guided search behavior, most likely because of the limited range of starting points used during training.

\section{EXPERIMENT 2}

Experiment 1 showed that the arena's landmarks were strongly involved in the consistent group's finding of the hidden food. Our next question was how these landmarks were used by the rats. In Experiment 2, we investigated how removing different numbers of landmarks influenced performance. Both groups were tested in sessions with zero, one, three, five, or all six landmarks removed from the arena.

We also examined one other potential source of useful spatial information that was present in the arena, the relative location of the hidden food itself. Although Experiment 1 had ruled out the food's odor's acting as a cue, the fixed relations between the baited poles themselves could have been used by the consistent group as an aid in locating the food. We investigated this possibility by examining, in conjunction with landmark removal, conditions in which the target poles were baited or not. If such spatial information from the baits were capable of being used by the consistent group, its performance should be better in baited rather than unbaited condi- 
tions. In contrast, the random group's performance should not differ between conditions, since its bait placements were randomized in each session.

\section{Method}

\section{Subjects and Apparatus}

After Experiment 1 and prior to this one, both groups of rats participated in 200 further sessions over a 4 -month period. During these sessions, they participated in a pilot experiment involving the use of multidimensional scaling for investigating animal spatial representation. These tests consisted primarily of baseline sessions, with occasional probe tests involving pairwise spatial transpositions of the arena's various landmarks. Over these sessions, the rats were started from five different locations $\left(45^{\circ}, 135^{\circ}, 225^{\circ}\right.$, $315^{\circ}$, and the center). The apparatus was the same as in Experiment 1 , except that we replaced the paper curtain surrounding the arena with beige vinyl shower curtains.

\section{Procedure}

We conducted four sessions on each day, two in the morning and two in the afternoon. The second and fourth sessions were conducted 60-90 min after the first and third, during which other rats were tested. The first three sessions of each day were identical to the training sessions of Experiment 1 . The fourth session tested one of nine test conditions. These test conditions consisted of having zero, one, three, five, or six of the landmarks removed from the arena, crossed with whether or not the six target poles were either baited with three pellets of food or not. The baited sixlandmark condition was not tested, because it was the same as the baseline session. For the purposes of statistical analysis, estimates for this condition were derived from average performance in the daily baseline sessions. We randomly determined for each rat and session the identities of the landmarks that were removed. Each rat received the nine test conditions in a random order in a 9-day block. Five starting locations were used $\left(45^{\circ}, 135^{\circ}, 225^{\circ}, 315^{\circ}\right.$, and center). They were block-randomized independently of the main experimental manipulation.

\section{Results and Discussion}

The number of landmarks and the presence of food on target poles both had significant influences on the consistent group's performance (see Figure 3). Mean choice efficiency became significantly poorer as increasing numbers of the landmarks were removed $[F(4,36)=6.5]$ and was significantly poorer for those sessions in which the target poles were not baited $[F(1,9)=22.8]$. Despite the apparent interaction in Figure 3, in which the effects of bait absence appear greatest as more of the landmarks were removed, this term was not significant in the ANOVA $(p<.09)$. A second ANOVA restricted to just the extreme landmark conditions (zero and one combined vs. six landmarks present) did reveal a significant interaction between these two factors $[F(2,8)=5.1]$; the influence of having the poles baited diminished as landmarks were placed back into the arena. Identical analyses of the random group's choice efficiency revealed no significant effects of either landmark removal or bait status on their performance (mean choice efficiency: baited condition = 13.6; unbaited condition $=13.9$ ).

This experiment revealed two additional facets of the consistent group's abilities to locate the hidden food in the arena. Whereas in Experiment 1 the landmarks were found to be important, in Experiment 2 the number of landmarks was found to be critical. Most, if not all, of the landmarks contributed to this performance; the rats' effectiveness at recovering the food was a direct function of the number of landmarks present.

Another influence revealed by Experiment 2 was the possibility that the spatial relations between the food locations themselves might also aid the consistent group's performance. This was suggested by the relative effec-

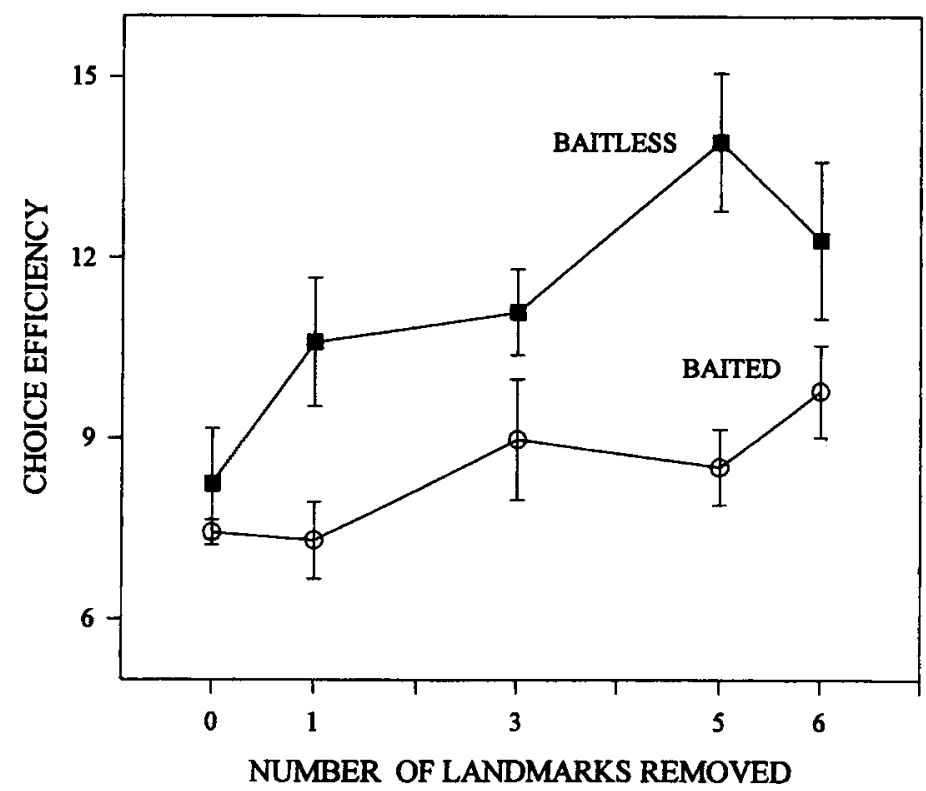

Figure 3. Mean choice efficiency scores for the consistent group in Experiment 2 as a function of the number of landmarks removed from the arena in both the presence and absence of baits. 
tiveness of the rats' search performance even in the absence of any landmarks as long as the poles were still baited. One obvious possibility is that the food's odor was somehow involved. This appears not to have been the case. First, the consistent group's performance was consistently better than the random group's in both experiments. Second, the random group's initial choices in a session were at chance with respect to the newly baited locations. Third, the presence and absence of food had no significant influence on the random group's performance in this experiment. Furthermore, our strong impression was that the rats only seemed to smell the food after rearing up to the lip of the food cup. All of these findings point to the conclusion that the food's odor per se was not contributing to the rats' performance.

Neither can these results be easily attributed to any search-disrupting "frustration" associated with not finding food in the arena for the first time. This seems ruled out by the absence of any bait effect in the random group and by the changing size of the effect as a function of the number of landmarks in the consistent group. In the latter case, why would these rats show the smallest effect of food absence, and the least "frustration," when all landmarks were present, which was the context most associated with food in the past?

The findings of Experiment 2 seem most consistent with the idea that the beneficial effect of the baited poles on search behavior stems from their stable locations relative to one another. This consistency allows a rat to search more effectively after initially finding some of the baited poles, perhaps by strengthening a "working hypothesis" about its current location and orientation within the arena. Of course, deriving useful spatial information from a succession of fixed, but hidden, point sources would be difficult without some frame of reference. Normally, the landmarks serve this function. But even without the landmarks, several sources of useful orienting information were still present in the arena. Among these were the geometric relations between the poles and the arena boundary (Cheng, 1986; Margules \& Gallistel, 1988; Williams, Barnett, \& Meck, 1990) and the fixed geometric relations of the poles themselves. Both of these global reference frames might have helped the rats to disambiguate their current spatial location. In experiments motivated by the present results, Brown and Terrinoni (1996) have recently confirmed that rats are capable of learning the pattern of hidden distributed food in a pole search task.

\section{EXPERIMENT 3}

Experiment 2 suggested that information was used simultaneously from multiple landmarks to locate the hidden food. What is the nature of this information? Gallistel (1990) and Cheng (1986) have argued that primarily the spatial configuration of the surrounding environment and landmarks are the most important in guiding animal spatial behavior. That is, not the featural qualities of the surrounding reference points but their geometric con- figuration is of most importance. Tests involving male rats have tended to confirm this possibility, showing that these rats preferentially encode the geometric properties of the surrounding environment and generally do not strongly attend to the local identity of landmarks in spatial discriminations (Cheng, 1986; Margules \& Gallistel, 1988; Williams et al., 1990; but see Suzuki et al., 1980). Contrary to this, evidence collected from experiments testing gerbils (Collett et al., 1986), hamsters (Poucet et al., 1986), pigeons (Spetch \& Edwards, 1988) and female rats (Williams et al., 1990) has suggested that these animals use global geometric cues and local landmark identity when both types of cues are available in spatial tasks.

Given our choice of male rats for exploring this new spatial task, we were now quite interested in seeing to what extent these rats were using the metric properties of the landmarks to guide their effective search performance. In addition, we were interested in testing whether small discrete proximate landmarks would be sufficient to generate the kind of geometric stimulus control normally exhibited by the overall shape of the testing environment (Cheng, 1986; Cheng \& Gallistel, 1984; Margules \& Gallistel, 1988; Williams et al., 1990). Thus, Experiment 3 was designed to assess whether such emergent geometric relations existed among the landmarks, and to what extent they controlled the behavior of the rats. In addition, we examined to what extent, if at all, the specific identities of the landmarks also entered into the control of the consistent group's search performance.

Experiment 3 consisted of three transfer tests. These examined the performance of the consistent group when the identity and geometric configuration of the landmarks were either the same as in training or altered systematically. If only the landmarks' geometric properties were important for performance, novel landmarks retaining the same geometry as previously experienced should allow for search at levels near that of baseline performance, and far more effectively than if the same landmarks were arranged in novel geometric configuration. In contrast, if the identity of the landmarks was crucial for navigation, novel landmarks should disrupt performance regardless of whether their configuration was the same as during training or not.

Each of the three transfer tests compared three conditions. The baseline condition involved testing the original six landmarks and configuration exactly as in the previous experiments. The training geometry condition retained the same spatial configuration of landmarks as used during training, but the identity of the individual landmarks was altered (see below). The novel geometry condition tested the same landmarks as used in the training geometry condition, but in completely new geometric configurations. Performance was expected to be best in the baseline condition. Correspondingly, the poorest performance was expected in the novel geometry condition, in which both the identity and the geometric properties of the landmarks were changed, neither of which could help with finding food. When compared with the critical training geometry condition, these two reference points help to reveal how 
the rats had encoded the visual and geometric properties of the landmarks. For instance, comparable performance in the training geometry and baseline conditions would suggest that the specifics of the landmarks were unimportant and that only their geometry was needed by the rats. Alternately, similar levels of performance in the training geometry and novel geometry conditions would suggest that landmark identity was most crucial to the rats. Finally, if performance in the training geometry condition fell in between these conditions, it would indicate that both landmark-specific identity and landmark-independent geometric properties were contributing to the animals' locating the baited poles.

Each test was designed to investigate a different alteration of the landmarks' stimulus properties. The conditions for each test are depicted in Figure 4. In the exchanged landmarks test, the effects of rearranging the familiar training landmarks were examined. In the novel landmarks test, six novel objects replaced the familiar landmarks. In the identical landmarks test, six identical landmarks were tested in place of the familiar landmarks.

\section{Method}

\section{Subjects and Apparatus}

Only the consistent group of the previous experiments was tested. The apparatus was the same as in Experiment 2.

\section{Procedure}

The daily procedure for these :ests was much like that in Experiment 2 . We tested rats four times each day, twice in the morning and twice in the afternoon. The first three sessions were all conducted like the previous training sessions. We performed the transfer tests in the fourth session. Five starting locations were used $\left(45^{\circ}, 135^{\circ}, 225^{\circ}, 315^{\circ}\right.$, and the center); they were randomized in blocks independently of the other experimental manipulations

Test 1: Exchanged landmarks. The exchanged landmarks test was conducted over 5 days. Each rat received five different test conditions during the fourth session of these test days. These conditions consisted of: (1) landmarks in training configuration, with target poles nat baited; (2-3) rearranged landmarks in the training configuration, with the target poles baited or not baited; (4-5) landmarks in a novel configuration, with target poles baited or not baited. We created 14 different novel landmark configurations for use in the novel geometry conditions of these tests. Two of these were selected randomly without replacement for each rat's individual tests. For the training geometry condition, the rearrangement of the landmarks was determined randomly for each condition and rat. Examples of these conditions are shown in the top part of Figure 4.

Test 2: Novel landmarks. The five conditions of the novel landmarks test were identical to the exchanged landmarks test, except that six novel objects were substituted for the original landmarks in Conditions 2, 3, 4, and 5. These six new objects were: a glass bottle, a plastic coffee filter, a standard pigeon food hopper, a coffee canister, a small box of tissues, and a small labeled can of tomato paste. We tested each rat in the novel geometry conditions with two novel landmark configurations not used in Test 1. Examples of these conditions are shown in the middle of Figure 4.

Test 3: Identical landmarks. The procedure for this test was identical to those above, except that we used six identical unopened $305-\mathrm{g}$ soup cans (labels removed) in the training and novel geometry conditions. Each can's seam was randomly oriented across sessions. Examples of these conditions are shown in the bottom section of Figure 4.

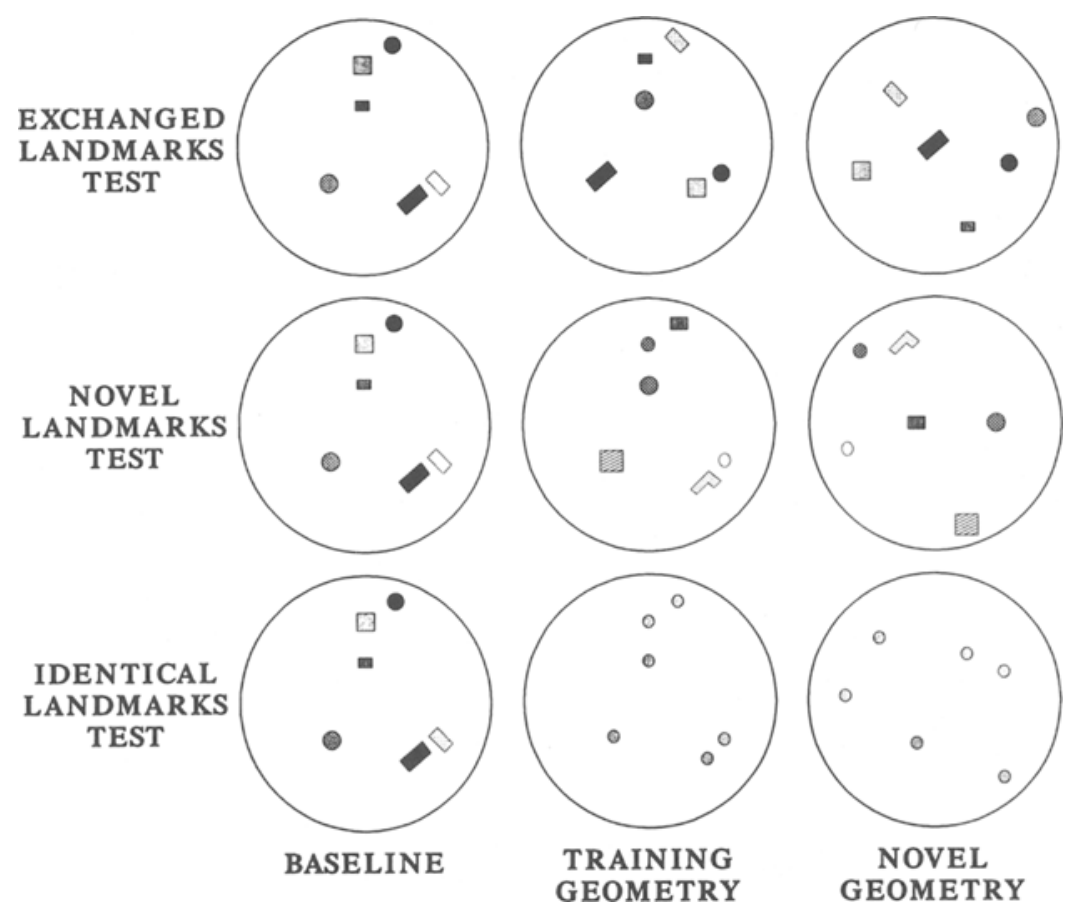

Figure 4. Configurations of landmarks for the exchanged, novel, and identical landmark transfer tests of Experiment 3. The landmark symbols in each test each represent different objects. 


\section{Results and Discussion}

The results from the exchanged, novel, and identical tests are shown in Figure 5, averaged across the baited and unbaited manipulation. The outcomes of all three tests were basically the same: choice efficiency was best in the baseline conditions (solid bars), poorest in novel geometry conditions (open bars), and intermediate to these in the training geometry condition (hatched bars).

\section{Exchanged Landmarks}

We used three repeated measures ANOVAs (condition $X$ bait presence) to compare choice efficiency among the different conditions. When compared with the baseline condition, the rats performed significantly poorer in both the training geometry $[F(1,9)=9.8]$ and novel geometry conditions $[F(1,9)=41.1]$. Choice efficiency was significantly better in the training geometry condition than in the novel geometry condition $[F(1,9)=11.8]$. We found no significant main effects or interactions of bait presence in any of the three ANOVAs, except when comparing baseline and training geometry conditions $[F(1,9)=8.6]$, where choice efficiency was reliably better in the baited condition than in the unbaited condition.

\section{Novel Landmarks}

Rats performed significantly poorer both in the training geometry $[F(1,9)=5.1]$ and in the novel geometry condition $[F(1,9)=14.9]$ than in the baseline condition. Choice efficiency was significantly better in the training geometry condition than in the novel geometry condition $[F(1,9)=8.1]$. No significant main effects or interactions with the presence or absence of food were found in any of the three ANOVAs.

\section{Identical Landmarks}

Compared with the baseline condition, choice efficiency was significantly poorer only in novel geometry conditions $[F(1,9)=9.2]$. As in the previous tests, performance was on the average poorer in the training geometry condition than in the baseline condition, but this difference was not statistically significant $[F(1,9)=$ $1.0]$. Performance in the training geometry condition was again significantly better than in the novel geometry condition $[F(1,9)=6.0]$. No significant main effects or interactions with the presence or absence of food were found in any of the ANOVAs.

Overall, Experiment 3 provided clear evidence that both the geometric relations among landmarks and the identity of the landmarks seemed to make important contributions to guiding the rats' search behavior. Regardless of whether the landmarks were exchanged, novel, or entirely identical, rats performed better when the geometric arrangement of these landmarks was the same as that experienced during training than they did with a novel configuration. The strongest demonstration of this occurred in the identical landmarks test, where the level of performance was statistically the same whether the original landmarks or identical landmarks formed the training configuration. The results from all three tests, however, suggest that the consistent group recognized and utilized spatial information derived from geometric relations among the landmarks. This conclusion is consistent with Experiment 2's finding that performance depended directly on the number of landmarks present in the arena. Collectively, these results indicate that small discrete proximate landmarks are indeed sufficient to generate the kind of geometric stimulus control more typically exhibited by the overall shape of the testing environment (Cheng, 1986; Cheng \& Gallistel, 1984; Margules \& Gallistel, 1988; Williams et al., 1990).

In addition to this geometric information, the results also indicate that rats had encoded the identity and positions of the training landmarks. This can be seen in the exchanged landmarks test, where during the baseline

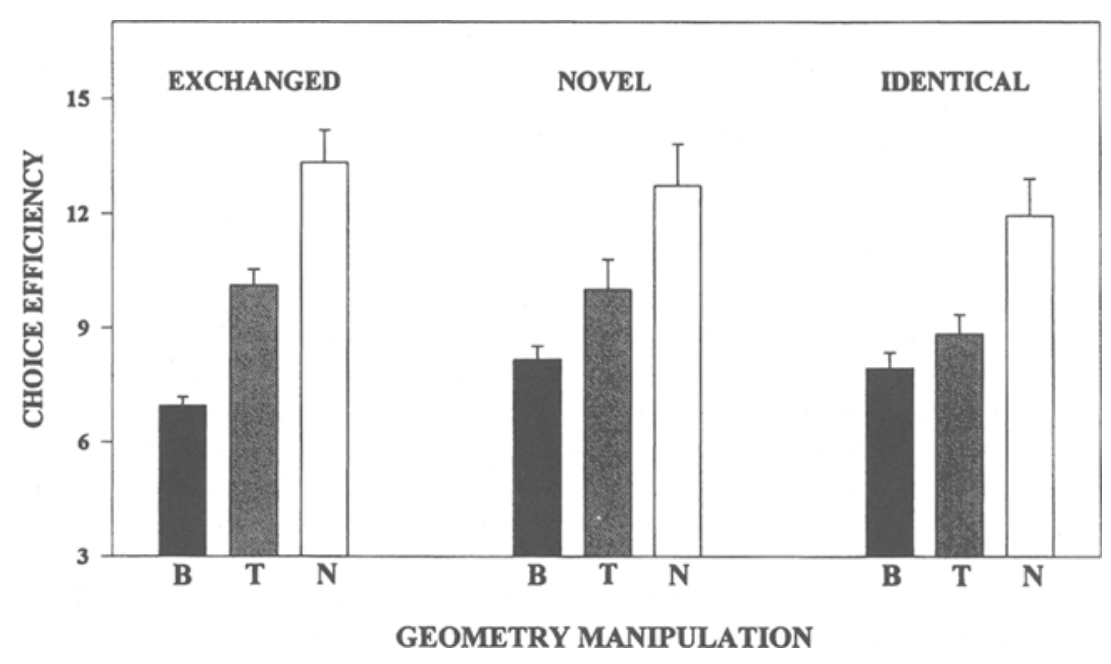

Figure 5. Mean choice efficiency for the consistent group in the baseline (B), training geometry (T), and novel geometry (N) test conditions of the exchanged, novel, and identical landmark transfer tests of Experiment 3. 
condition the rats performed better than in the training geometry condition, despite the latter condition's retention of the familiar landmarks and geometry. As such, these results resemble the deficits found by Suzuki et al. (1980) when they similarly transposed extra-maze stimuli in the radial maze.

The conclusions that these male rats encoded both landmark geometry and identity extends the results of previous studies concerning rat spatial memory. Cheng (1986) and Margules and Gallistel (1988) have argued that the metric relations of the apparatus' shape and goal locations are primarily used by rats, and that the identity of local parts within this "environmental shape" have only a secondary impact on performance. Although Cheng (1986) considered the possibility that some featural information associated with goal locations may be encoded within the context of a "geometric module," he did not suggest that featural information of distal landmarks was important, which is suggested by the results of Experiments 2 and 3. Williams et al. (1990) suggested that this geometric bias was characteristic only of male rats, and that female rats relied upon both landmark identity and environmental shape to locate goals. Clearly this is not the case. In contrast with the findings of Williams et al., our results suggest that landmark geometry and identity are also important for accurate performance by male rats. These apparently contradictory results may be due to differences in the tasks employed. We utilized an open-field task in which the animal moved through a landmark-filled environment, whereas Williams et al. used a radial maze task that did not allow the rats to move among landmarks. Perhaps male rats can only encode the identity of highly proximal landmarks in their immediate surroundings.

Finally, although the landmark manipulations of Experiment 3 are consistent with the results of Experiment 2, the presence or absence of food during the transfer tests showed little consistent effect on search performance in contrast to what was found in Experiment 2. The absence of such effects in the present test, and its apparent dependence in Experiment 2 on the absence of landmarks in the arena, suggests that whatever information is derived from the configuration of baited poles is easily overshadowed by the featural and geometric information of the landmarks.

\section{GENERAL DISCUSSION}

Over the three experiments, we found that the rats in the consistent group located the arena's multiple goal locations by means of the intra-arena landmarks (Experiment 1); that most, if not all, of the landmarks contributed to the success of their search (Experiment 2); and that both the identity and the relative geometry of the intra-arena landmarks were coded and used by the rats (Experiment 3). Besides the development of a new spatial task for testing animals, the most important new results from these experiments concerned the clear evi- dence that male rats code both landmark identity and geometry in order to navigate their way through this spatial task, and that the coding of global geometry by rats can be derived from discrete proximal landmarks, as well as from overall environmental shape as previously demonstrated. Although evidence of the former has been found in other species (Cheng, 1989; Collett et al., 1986; Poucet et al., 1986), surprisingly little direct evidence for this fact has been actually offered for rats, since previous research has tended to emphasize only the role of the structural geometry of the testing apparatus in the control of rat spatial behavior (Cheng, 1986; Gallistel, 1990; Margules \& Gallistel, 1988; Williams et al., 1990).

Besides showing the importance of the landmark identity and arrangement, these experiments further attest to the strong propensity of rats to redundantly use multiple cues and strategies to navigate about a space. It appears that rats will exploit multiple types of information to successfully recover spatially distributed food. For instance, the results from Experiment 1 suggested that the rats may have initially utilized some limited form of learned response-based strategy in addition to their spatial use of the landmarks. Even the random group, which searched for inconsistently located rewards, was able to improve its performance by learning to reduce pole revisits within a session. Experiment 2 (but not Experiment 3 ) suggested that the rats may have been able to use information derived from the spatial relations among the baited poles themselves. Collectively, such findings indicate the paramount need for the careful control and evaluation of any spatial task's discriminative cues, especially prior to making claims, for example, about the processes affected by physiological or pharmacological interventions in such tasks.

Toward this general goal, the present open-field task offers several advantages over previous procedures used to study spatial behavior in animals. In an open-field task, the animal's choice behavior is not unduly restricted by the physical structure of the apparatus, as is the case in the radial maze. This allows the animal to more directly express its spatial knowledge. Furthermore, since the landmarks are placed within the animal's environment, the present situation mimics far better the structure of the natural world than do the vast majority of spatial tasks and mazes. In addition, the task also allows for the precise control over the stimuli available to the animal, permitting the systematic investigation of the visual and spatial properties controlling search behavior. Finally, the presence of multiple "distractor" poles allows for the clear, easy, and precise measurement of an animal's choice errors, unlike the case with the water maze or open-field digging tasks (e.g., Cheng, 1989; Morris, 1981). We believe that these three experiments show the promise and utility of this cue-controlled arena and its search task for investigating the representation of space by animals, revealing in the present case evidence that male rats can encode both landmark identity and geometric information 
about local landmarks in their representation of spatial environments.

\section{REFERENCES}

Brown, M. F. (1992). Does a cognitive map guide choices in radialarm maze? Journal of Experimental Psychology: Animal Behavior Processes, 18, 56-66.

Brown, M. F., \& TERrinoni, M. (1996). Control of choice by the spatial configuration of goals. Journal of Experimental Psychology: Animal Behavior Processes, 4, 438-446.

Cartwright, B. A., \& Collett, T. S. (1983). Landmark learning in bees. Journal of Comparative Physiology, 151, 521-543.

CHENG, K. (1986). A purely geometric module in the rat's spatial representation. Cognition, 23, 149-178.

Cheng, K. (1989). The vector sum model of pigeon landmark use Journal of Experimental Psychology: Animal Behavior Processes, 15, 366-375.

CHENG, K. (1994). The determination of direction in landmark-based spatial search in pigeons: A further test of the vector sum model. $A n-$ imal Learning \& Motivation, 22, 291-301.

Cheng, K., Collett, T. S., Pickhard, A., \& Wehner, R. (1987). The use of visual landmarks by honey bees: Bees weight landmarks according to their distance from the goal. Journal of Comparative Physiology, 161, 469-475.

Cheng, K., \& Gallistel, C. R. (1984). Testing the geometric power of an animal's spatial representation. In H. L. Roitblat, T. G. Bever, \& H. S. Terrace (Eds.), Animal cognition (pp. 409-423). Hillsdale, NJ: Erlbaum.

Collett, T. S., Cartwright, B. A., \& Smith, B. A. (1986). Landmark learning and visuo-spatial memories in gerbils. Journal of Comparative Physiology, 158, 835-851.

Cook, R. G. (1993). The experimental analysis of cognition in animals. Psychological Science, 4, 174-178.

Cook, R. G., \& BRowN, M. F. (1985). Retroactive interference in rat radial maze performance: The role of point of delay interpolation and the similarity and amount of interpolated material. Animal Learning \& Behavior, 13, 116-120.

COOK, R. G., BRowN, M. F., \& RILEY, D. A. (1985). Flexible memory processing in rats: The use of prospective and retrospective information in the radial arm maze. Journal of Experimental Psychology: Animal Behavior Processes, 11, 453-469.

Gallistel, C. R. (1990). The organization of learning. Cambridge, MA: MIT Press.

GouLd, J. L. (1986). The locale map of honey bees: Do insects have cognitive maps? Science, 232, 861-863.

Kraemer, P. J., Gilbert, M. E., \& INNIS, N. K. (1983). The influence of cue type and configuration upon radial-maze performance in the rat. Animal Learning \& Behavior, 11, 373-380.

LEONARD, B., \& MCNAUGHTON, B. L. (1990). Spatial representation in rat: Conceptual, behavioral, and neurophysiological perspectives. In R. P. Kesner \& D. S. Olton (Eds.), Neurobiology of comparative cognition (pp. 363-422). Hillsdale, NJ: Erlbaum.

Margules, J., \& Gallistel, C. R. (1988). Heading in the rat: Determination by environmental shape. Animal Learning \& Behavior, 16, 404-410.

Menzel, E. W. (1973). Chimpanzee spatial memory organization. Science, 182, 943-945.

MoRRIS, R. G. M. (1981). Spatial localization does not require the presence of local cues. Learning \& Motivation, 12, 239-261.

OLTON, D. S. (1978). Characteristics of spatial memory. In S. H. Hulse, H. Fowler, \& W. K. Honig (Eds.), Cognitive processes in animal behavior (pp. 341-373). Hillsdale, NJ: Erlbaum.

Olton, D. S., \& SAmuelson, R. J. (1976). Remembrance of places past: Spatial memory in rats. Journal of Experimental Psychology: Animal Behavior Processes, 2, 97-116.

Olton, D. S., \& SCHLOSBERG, P. (1978). Food-searching strategies in young rats: Win-shift predominates over win-stay. Journal of Comparative \& Physiological Psychology, 92, 609-618.

PEARCE, J. M. (1987). Introduction to animal cognition. Hillsdale, NJ: Erlbaum.

Poucet, B., Chapuis, N., Durup, M., \& Thinus-Blanc, C. (1986). A study of exploratory behavior as an index of spatial knowledge in hamsters. Animal Learning \& Behavior, 14, 93-100.

SPETCH, M. L., \& EDWARDS, C. A. (1988). Pigeons', Columba livia, use of global and local cues for spatial memory. Animal Behaviour, 36 , 293-296.

Suzuki, S., Augerinos, G., \& Black, A. H. (1980). Stimulus control of spatial behavior on eight-arm radial maze in rats. Learning \& $\mathrm{Mo}$ tivation, 11, 1-18.

TINKLEPAUGH, O. L. (1932). Multiple delayed reaction with chimpanzee and monkeys. Journal of Comparative Psychology, 13, 207243.

Tolman, E. C., Ritchie, B. F., \& Kalish, D. (1946). Studies in spatial learning: I. Orientation and the short-cut. Journal of Experimental Psychology, 36, 13-24

Williams, C. L., BarnetT, A. M., \& MECK, W. H. (1990). Organizational effects of early gonadal secretions on sexual differentiation in spatial memory. Behavioral Neuroscience, 104, 84-97.

ZOLADEK, L., \& RORERTS, W. A. (1978). The sensory basis of spatial memory in the rat. Animal Learning \& Behavior, 6, 77-81.

(Manuscript received February 13, 1996; revision accepted for publication October $31,1996$. 